\title{
The cell membrane
}

\section{Anamourlis iD}

Department of Anaesthesia, Chris Hani Baragwanath Academic Hospital, School of Clinical Medicine, Faculty of Health Sciences, University of the Witwatersrand, South Africa

Corresponding author, email: chris.anamourlis@gmail.com

\section{Summary}

The cell membrane forms an integral part of cellular homeostasis. This article will review the structure and function of physiological membranes and how the lipid bilayer plays an important role in maintaining cellular integrity, ion permeability, and membrane potential as well as being selectively permeable to certain biological substances. This review looks at the physico-chemical and structural properties of the plasma membrane which affords it the unique property of selective permeability and action potential generation. Furthermore, the mechanisms by which permeability occurs across the membrane are also discussed.

Keywords: cell membrane, cellular homeostasis, lipid bilayer

\section{Structure and composition}

Plasma membranes are extremely pliable structures that are composed of a phospholipid bilayer that separates the intracellular and extracellular fluid compartments. Cholesterol is a key component that contributes to the fluidity of the membrane, with various proteins imbedded within the membrane conferring a variety of specific functions. One phospholipid molecule has a head and a tail. The head is comprised of a hydrophilic (negatively charged) phosphate group and has two side-by-side fatty acid chains which are known as the tail (Figure 1). The polarised head allows for the interaction of this portion of the bilayer with

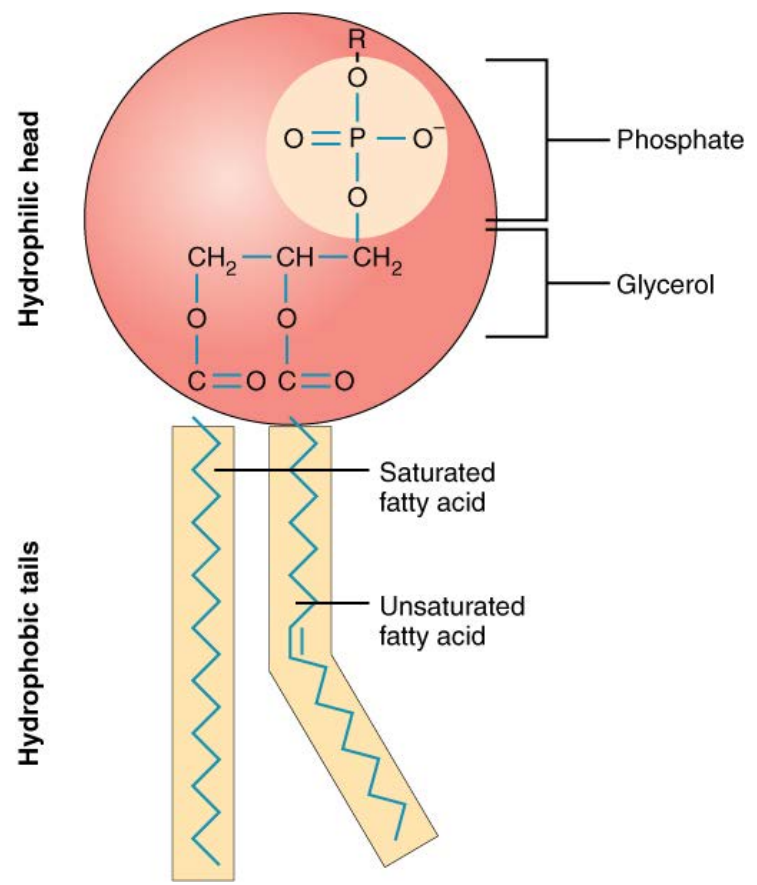

Figure 1: Phospholipid structure

A phospholipid molecule consists of a polar phosphate "head," which is hydrophilic and a non-polar lipid "tail," which is hydrophobic. Unsaturated fatty acids result in kinks in the hydrophobic tails. ${ }^{1}$ water, both on the intracellular and extracellular aspect of the membrane. This contrasts with the lipid tails that are uncharged, or non-polar, and are therefore hydrophobic. The combination of saturated and unsaturated fatty acid tails also contributes to the fluidity of this membrane. Therefore, the phospholipids that comprise the bilayer are known as amphipathic. ${ }^{1,2}$

The phospholipid bilayer is organised such that the layers are not only adjacent but the lipid tail portion of the molecules face each other inwards (Figure 2). Therefore, the heads face outwards with one layer exposed to the intracellular part of the cell, and another layer the exterior. This allows for the internal hydrophobic portion of the bilayer to be excluded from the intracellular and extracellular compartments. Membrane proteins and other fats, like cholesterol, traverse this space too, and provide additional functions to the cell membrane adding to cell homeostasis but do not compromise the fluid nature of the bilayer because of how they 'float' in or across this membrane. ${ }^{1-3}$

These physicochemical properties confer the characteristic of selective permeability of the cell membrane. The size and

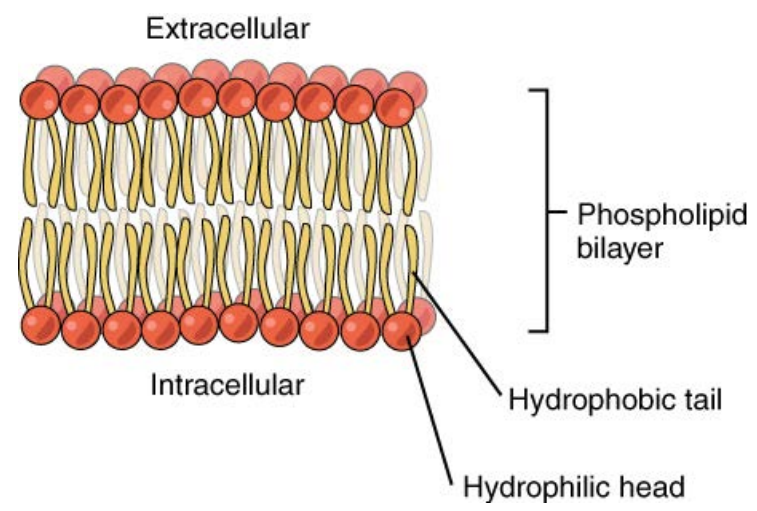

Figure 2: Phospholipid bilayer

The phospholipid bilayer consists of two adjacent sheets of phospholipids, arranged tail to tail. The hydrophobic tails associate with one another, forming the interior of the membrane. The polar heads contact the fluid inside and outside of the cell. ${ }^{1}$ 


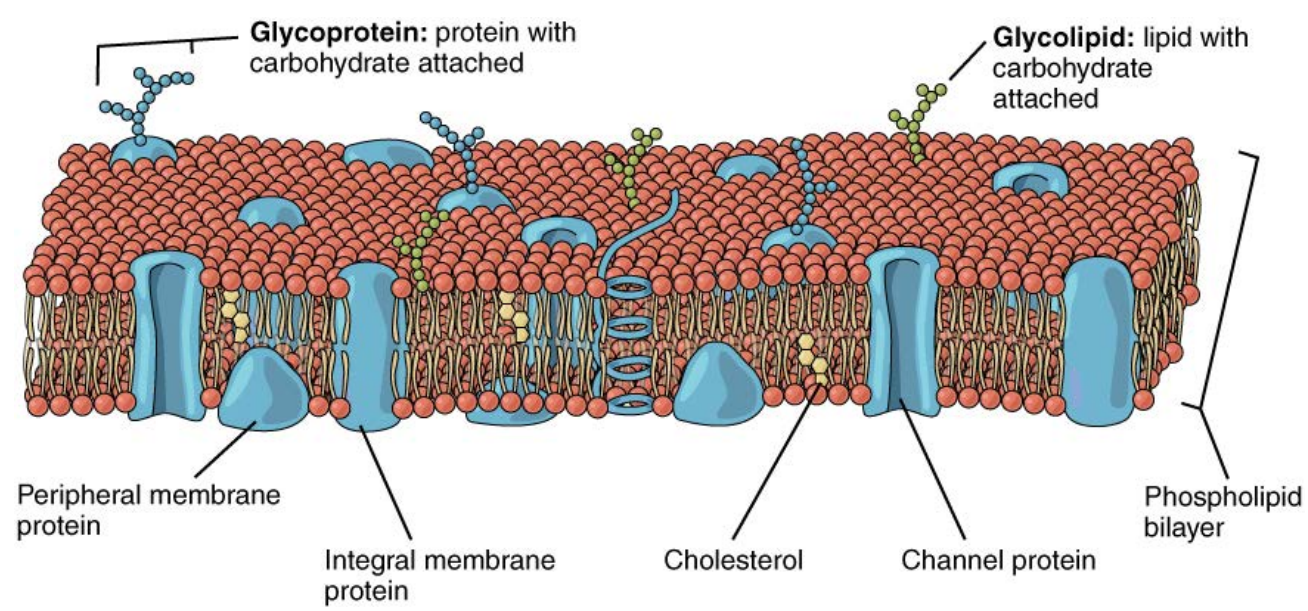

Figure 3: Cell membrane

The cell membrane is a phospholipid bilayer containing many different molecular components, including proteins and cholesterol, some with carbohydrate groups attached. ${ }^{1}$

solubility of the material determines whether or not the material is able to pass through the membrane. Oxygen and carbon dioxide can readily pass through, as well as lipid soluble substances. Water soluble glucose, and other charged molecules such as ions are unable to freely pass through and therefore require transmembrane proteins or channels in order to pass in or out of the cell.'

\section{Membrane proteins}

The plasma membrane has a variety of interspersed proteins within its lipid bilayer, mainly integral proteins and peripheral proteins. Integral proteins are embedded within the membrane (Figure 3). ${ }^{1}$

Examples of integral proteins are:

- Channel or transport proteins: these selectively allow materials, such as ions, through the plasma membrane.

- Receptors: also known as cell recognition proteins that allow for the selective binding of molecules outside of the cell. Binding usually induces a chemical reaction withing the cell through second messenger systems. These receptors are also like labels for the cell in order for cells to be recognised by other cells and proteins.

- Glycoproteins: these proteins have carbohydrate groups attached to them which extend into the extracellular matrix. This also aids in cell to cell recognition as the carbohydrates have 'tags' on them. They also form part of a very important system know as the glycocalyx which integrates extracellular membrane lipids as well. ${ }^{1}$

The glycocalyx has many roles that include:

- Cell-to-cell binding

- Receptors for hormone binding

- Enzymatic breakdown of nutrients

Enzymes also occur in the inner and outer portion of the cell membrane and are examples of peripheral cell proteins. Their functions are typically quite cell specific, for example in the intestinal tract where the extracellular peripheral proteins digest nutrients. ${ }^{1}$

\section{Membrane specialisations}

The membranes of specialised cells have unique functions conferred by components of the plasma membrane:

Microvilli are the finger-like projections of the cell membrane that increase the surface area of the cell in order to optimise absorption. They are found in cells of the small intestine.

Junctions (Figure 4) are a specialised group of proteins that tightly pack/connect cells together. There are three main types:

- Tight junctions: hold adjacent cells tightly together so that nothing can move between cells.

- Desmosomes: these are also called anchoring junctions and hold cells together by fibres which allow some movement without separation.

- Gap junctions: these are open areas within the plasma membrane between two cells and such proteins allow for the passage of certain chemicals between cells. ${ }^{1}$

\section{Ion permeability}

Since the cell membrane is semipermeable, it allows for a potential difference across the membrane because of selective ion transport and therefore different concentrations on either side of the membrane (electrical charge). The bilayer therefore also acts as a capacitor since there is the potential for an alteration in charge. Cells generally have a resting membrane potential 70-80 mV lower than extracellular fluid. ${ }^{2-4}$

The cell is permeable to potassium with potassium flowing freely out of the cell down a concentration gradient. This makes the resting membrane potential more negative which further opposes more potassium moving out of the cell establishing an equilibrium between the electrical and chemical gradients. ${ }^{1}$

The cell is impermeable to other cations; however, $\mathrm{Na}^{+}$does slowly leak down its concentration gradient. ${ }^{4}$ 

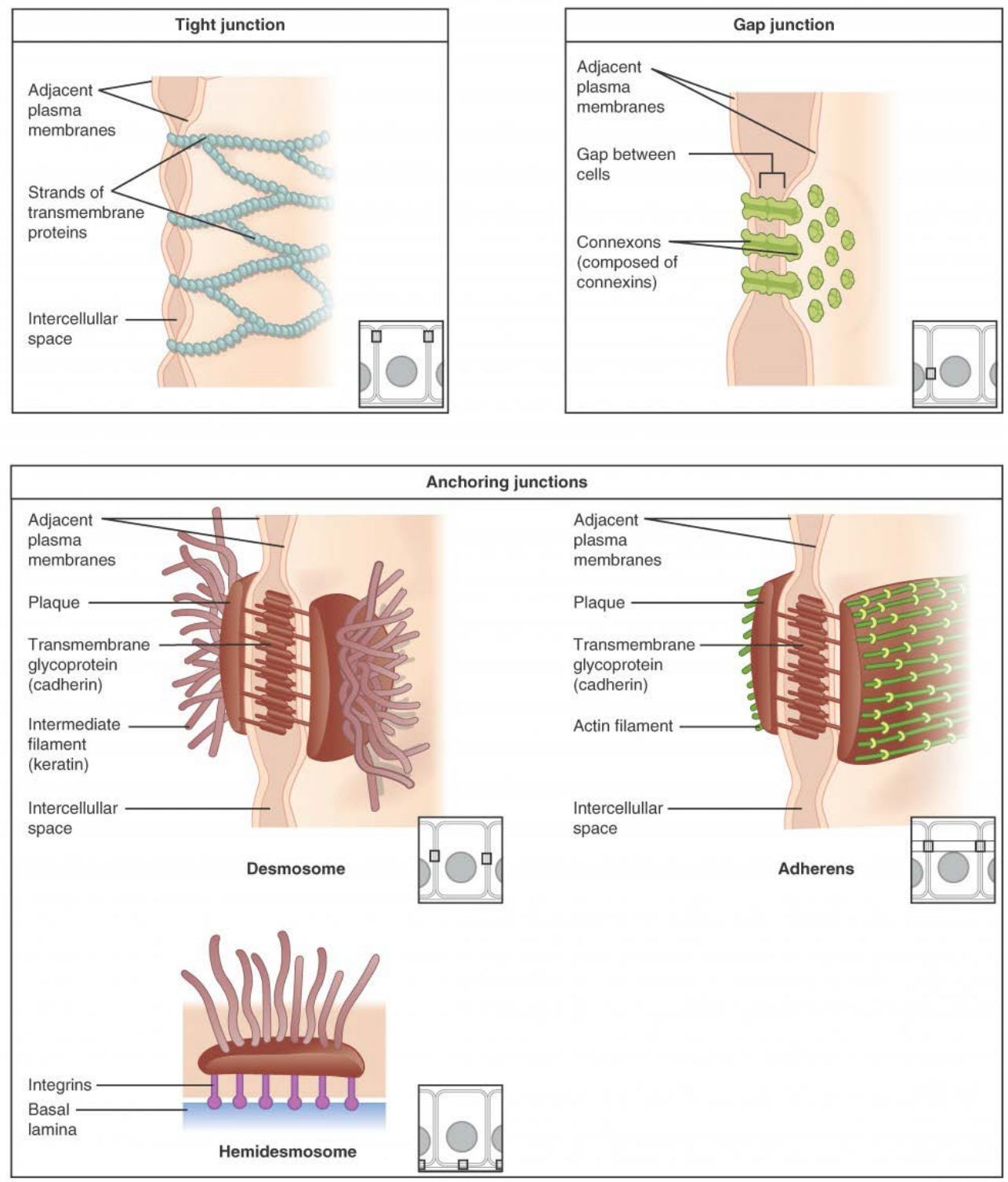

Figure 4: Three major types of cellular junctions ${ }^{1}$

The electrochemical gradient is established by the $\mathrm{NA}^{+} / \mathrm{K}^{+}-$ ATPase pump which is in essence electrogenic due to the unequal exchange of ions:

- three sodium ions are pumped out of the cell, and

- Two potassium ions are pumped into the cell. ${ }^{4}$

\begin{tabular}{ccc} 
Ion & Intracellular & Extracellular \\
\hline $\mathrm{Na}$ & 15 & 140 \\
$\mathrm{~K}$ & 150 & 4.5 \\
$\mathrm{Cl}$ & 10 & 100 \\
\hline
\end{tabular}

\section{Membrane potential}

As highlighted above, the resting membrane potential is generated when the diffusion of permeable potassium is opposed by the negative charge generated by its movement along its own diffusion gradient, and an equilibrium state is reached when these two opposing forces are equal. ${ }^{1-4}$

The potential difference generated by a permeable ion can be calculated using the Nernst equation, and assumes that the ion in question is not only permeable, but present in differing concentrations across a membrane in electrochemical equilibrium.

Nernst equation

$$
E(m V)=\frac{R T}{z . F} \ln \frac{[\text { ion]outside }}{[\text { ion] inside }}
$$

Where:

- $E$ is the equilibrium potential for the ion

- $R$ is the gas constant (8.314 J.deg-1.mol-1)

- $T$ is the temperature in Kelvin

- $F$ is Faradays constant

- $Z$ is the ionic valency (e.g. +2 for $M g,-1$ for $\mathrm{Cl}$ )

Goldman-Hodgkin-Katz equation

If the Nernst equation describes the potential difference of one permeable ion across a membrane, the actual membrane potential needs to be calculated by taking into consideration all 
ions of varying permeabilities across the same membrane. This is achieved with the Goldman-Hodgkin-Katz equation. ${ }^{3-5}$

$\mathrm{V}_{\mathrm{m}} \approx-60 \mathrm{mV} \log _{10} \frac{P_{K}[\mathrm{~K}]_{\text {in }}+P_{\mathrm{Na}}[\mathrm{Na}]_{\text {in }}+P_{\mathrm{Cl}}[\mathrm{Cl}]_{\text {out }}}{P_{\mathrm{K}}[\mathrm{K}]_{\text {out }}+P_{\mathrm{Na}}[\mathrm{Na}]_{\text {out }}+P_{\mathrm{Cl}}[\mathrm{Cl}]_{\text {in }}}$

Where,

- Px is the permeability constant for the ion $x$

- If the membrane is impermeable to $x$ then, $P x=0$

It is important to note that this equation, unlike the Nernst equation, does not take into account valency and that the concentration of negative ions is reversed compared to positive ones. $^{4}$

\section{Action potentials}

Excitable cells respond to stimuli by changing their membrane potential which is mediated either chemically (acetycholine receptors causing $\mathrm{Na}^{+}$channels to open) or physically (pressure receptors deforming $\mathrm{Na}^{+}$channels). Therefore, stimulating the excitable cell causes $\mathrm{Na}^{+}$permeability to increase which ultimately increases the membrane potential (makes it more negative). If the threshold potential is reached, then an action potential is generated. Fast $\mathrm{Na}^{+}$channels, known as voltagegated $\mathrm{Na}^{+}$channels, open when this threshold is reached (typically $-55 \mathrm{mV}$ ). This generates an all or nothing effect. ${ }^{1,3-6}$

The key players in cell membranes in the generation of an action potential are sodium and potassium.

The $\mathrm{Na}^{+}$channels that are responsible for depolarisation exist in three states:

1. Closed, impermeable to $\mathrm{Na}^{+}$.

2. Open, permeable to $\mathrm{Na}^{+}$, which occurs when the threshold potential is reached.

3. Inactivated, impermeable to $\mathrm{Na}^{+}$, which occurs shortly after the open state and lasts until the potential falls below $-50 \mathrm{mV} .^{1}$
Voltage gated $\mathrm{K}^{+}$channels are vital for repolarisation and open slowly with depolarisation thus increasing the potassium permeability and reducing the membrane potential. ${ }^{1}$

The action potential is propagated by an increase in sodium in one region that then diffuses down the cell membrane raising the membrane potential above the resting potential in adjacent cells. Fast $\mathrm{Na}^{+}$channels then open and the cell depolarises propagating a wave of depolarisation and repolarisation. This process highlights the significance of the sodium-potassium ATPase pump within cell membranes and the maintenance of cell membrane potentials. ${ }^{4,6}$

\section{Transport across cell membrane}

The cell membrane has the ability to regulate the concentration of substances inside the cell. lons such as $\mathrm{Na}^{+}, \mathrm{K}^{+}, \mathrm{Ca}^{+2}$, and $\mathrm{Cl}^{-}$ are maintained in homeostatic balance by the cell membrane along with sugars, fatty acids, amino acids, and waste products, especially carbon dioxide. The phospholipid bilayer offers the first level of control since the phospholipids are packed tightly together and the interior is hydrophobic. This structure is what confers the property of selective permeability. Substances meeting the criteria for selective permeability are able to cross the membrane unaided, that is, small, non-polar substances (e.g. oxygen, carbon monoxide, lipids and alcohol). Water soluble materials, however, need assistance passing through the membrane as they are repelled by the hydrophobic phospholipid tails. ${ }^{1,4,5,7}$

Movement across the membrane bilayer is generally classified as being either passive or active.

\section{Passive transport}

This form of transport uses concentration gradients and diffusion, whereby molecules will simply move down their gradients to areas of less concentration.

Several types of diffusion exist across the cell membrane, and these include:

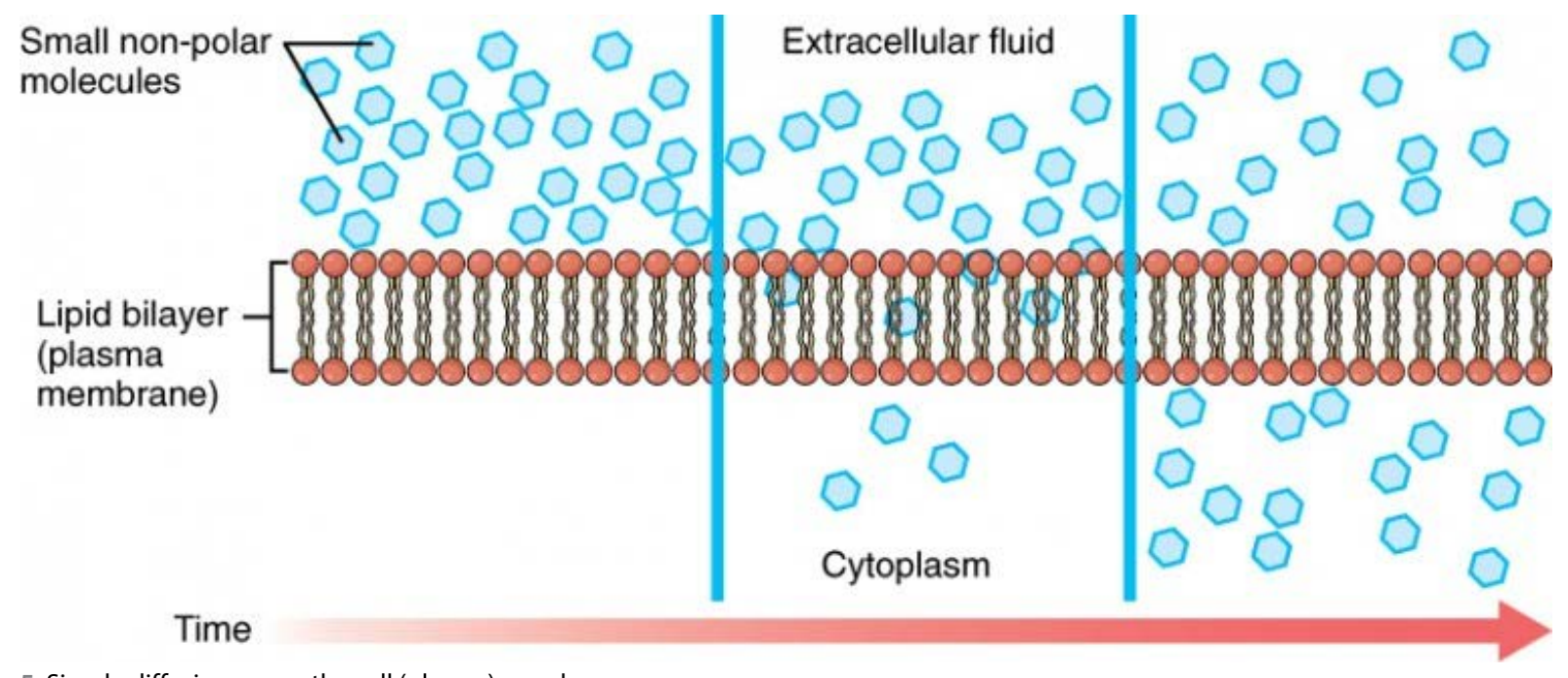


- Simple diffusion

- Osmosis

- Facilitated diffusion (uniporters)

The rate and extent of diffusion is affected by the gradients created by hydrostatic pressure, concentration of solutes as well as electrical charge. ${ }^{1,4}$

\section{Simple diffusion}

Any substance that has a concentration gradient across a membrane has the potential for simple diffusion, but that movement is dependant on the permeability of the membrane to that substance. Examples of substances that move easily are gases such as carbon dioxide and oxygen. Typically, oxygen is utilised rapidly by the cell and hence has much lower concentrations intracellularly, therefore creating a concentration gradient allowing constant diffusion into the cell. The converse is true for carbon dioxide, with high intracellular levels moving from the cytoplasm to the interstitial fluid compartment where the concentration is lower (Figure 5). ${ }^{1}$

\section{Osmosis}

Osmosis is the diffusion of water through a semipermeable membrane (Figure 6). Water is an example of a substance that can move freely across all cell membranes by moving through protein channels or by slipping between the lipid tails of the membrane itself. The movement of water is dependant on the solute concentration within it and therefore the concentration gradient created across membranes, etc. ${ }^{1}$

The osmotic pressure that is created by the solutes in solution are essentially what pulls water, as depicted by the figure below, with osmosis occurring when there is an imbalance of solutes on one side of the cellular membrane. The effect is concentration dependant with a greater osmotic pressure being created the more concentrated the solution. This effect is critical in maintaining homeostasis of the cellular environment such that isotonicity is achieved to ensure cellular functional integrity as well as structure. The kidneys are key players in maintaining homeostasis. ${ }^{1}$

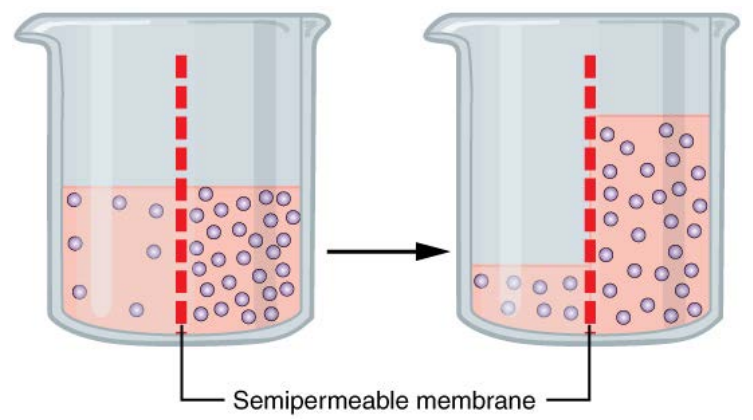

Figure 6: Osmosis

Osmosis is the diffusion of water through a semipermeable membrane down its concentration gradient. If a membrane is permeable to water, though not to a solute, water will equalise its own concentration by diffusing to the side of lower water concentration (and thus the side of higher solute concentration). In the beaker on the left, the solution on the right side of the membrane is hypertonic.' (a)
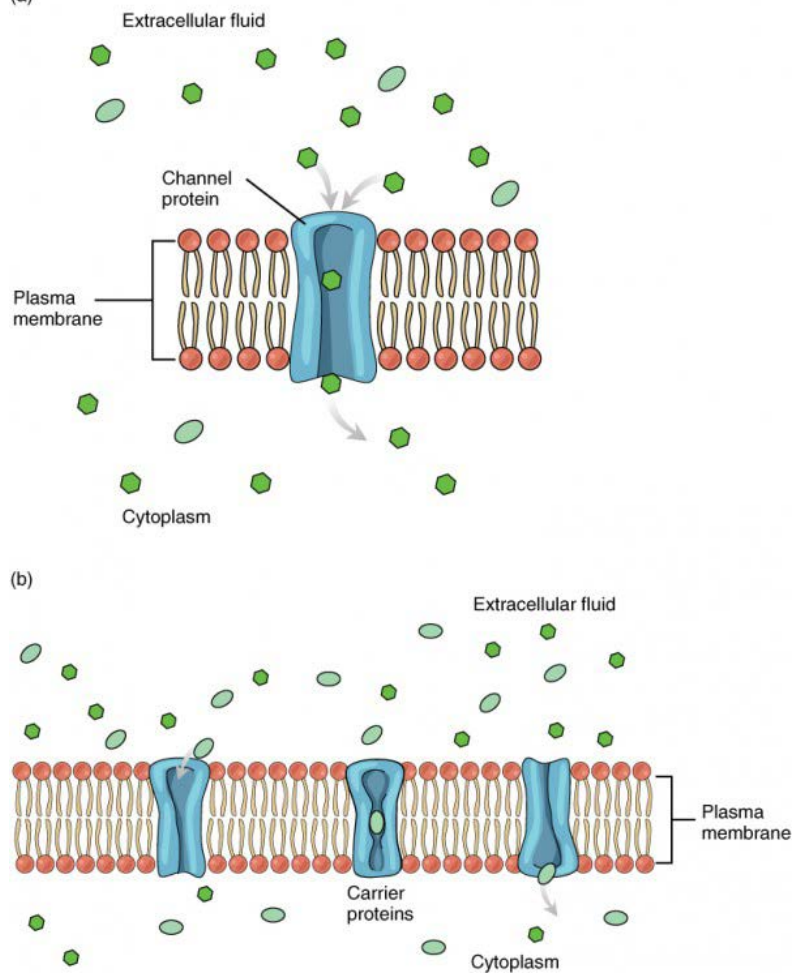

Figure 7: Facilitated diffusion

(a) Facilitated diffusion of substances crossing the cell (plasma) membrane takes place with the help of proteins such as channel proteins and carrier proteins.

Channel proteins are less selective than carrier proteins, and usually mildly

discriminate between their cargo based on size and charge. (b) Carrier proteins are more selective, often only allowing one particular type of molecule to cross.

\section{Facilitated diffusion}

Substances that cannot cross the cell membrane due to their physico-chemical properties (shape, size, polarity, etc.) require help crossing the membrane. Glucose is a common example where facilitated diffusion is necessary to allow the ATP generating substance passage into the cell. Despite a concentration gradient, the glucose molecule cannot cross the membrane unassisted, since it is a large polar molecule; therefore a specialised carrier protein called the glucose transporter is required for facilitated inward diffusion (Figure 7). Amino acids, and some metabolic waste products also require such carrier proteins for their movement. Importantly, facilitated diffusion is a passive process and therefore is not ATP consuming. ${ }^{1,4,5,7}$

\section{Active transport}

Substances that move against a concentration gradient require active transport which necessitates the use of energy in the form of ATP. Active transport mechanisms include:

- Primary active transport: the substance itself is moved.

- Secondary active transport: substances move down their electrochemical gradient by being coupled to another ion's active movement against a concentration gradient. Typically, sodium and hydrogen ions are examples.

- Co-transporters (symporters): carrier proteins that move two substances across a membrane (e.g. sodium and amino acids), whereby the process will be passive if the energy required to move one substance down its concentration gradient is 


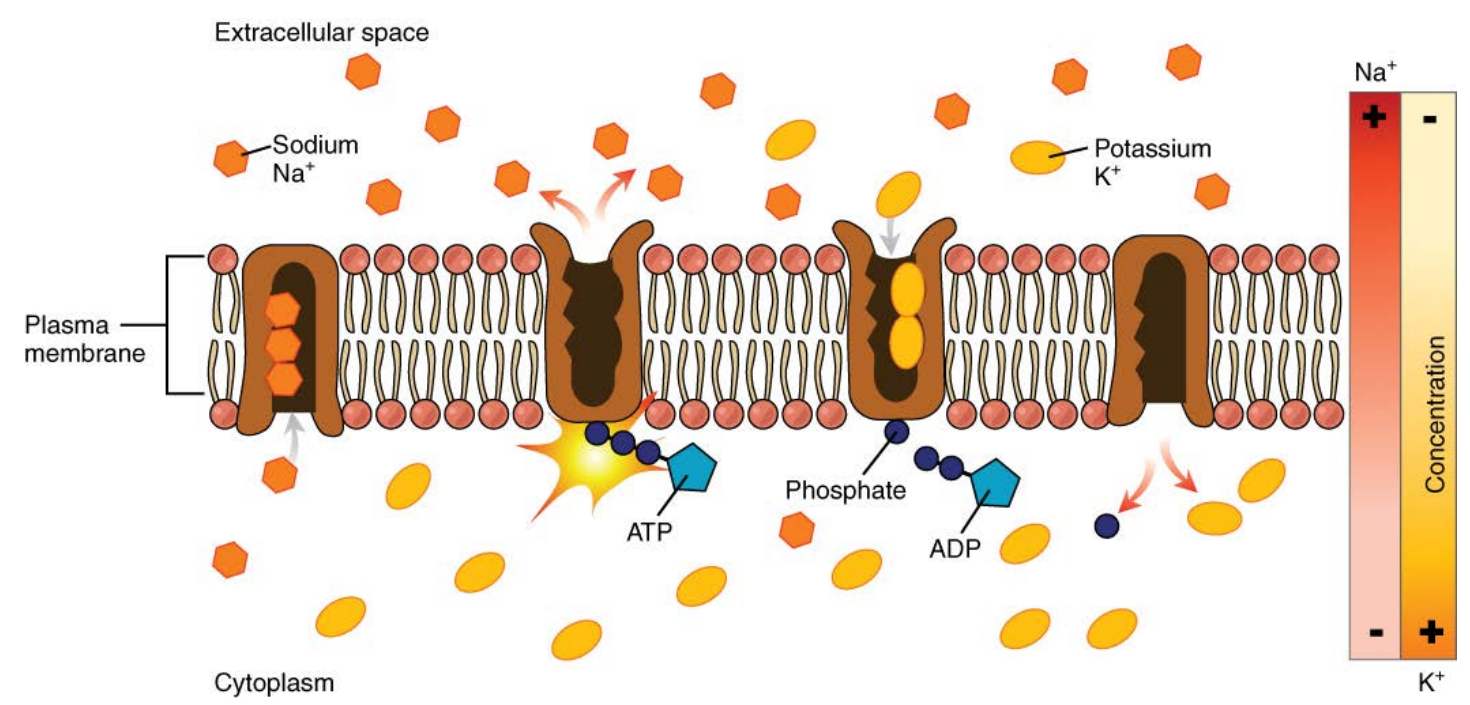

Figure 8: Sodium-potassium pump

The sodium-potassium pump is found in many cell (plasma) membranes. Powered by ATP, the pump moves sodium and potassium ions in opposite directions, each against its concentration gradient. In a single cycle of the pump, three sodium ions are extruded from and two potassium ions are imported into the cell. ${ }^{1}$

greater than the energy required to move the other substance up its concentration gradient.

- Counter-transporters (antiporters): two substances are moved in opposite directions by the use of carrier proteins. $^{1}$

The most common example of active transport in cells is that of the sodium-potassium pump, also known as $\mathrm{Na}^{+} / \mathrm{K}^{+}$ATPase (Figure 8). This pump is found in the membranes of many types of cells and is responsible for transporting three sodium ions out of the cell while moving two potassium into cells. This pump is found in abundance in nerve cells where it serves to maintain an electrical gradient across the nerve cell membrane. This electrical charge difference is responsible for establishing a relatively negative intracellular charge $(-70 \mathrm{mV})$ which is extremely important for nerve cell function, and hence accounts for the majority of the tissues ATP use. ${ }^{1,4,7,8}$

This pump also plays a role in cellular homeostasis as it has many other functions, including:

- Maintenance of cell volume by net loss of osmoles.

- Maintenance of the potential difference across the membrane as mentioned in the above example.

- Establishing chemical gradients to be used in secondary active transport mechanisms, e.g. S-GLUT transporter. ${ }^{4}$

\section{Endocytosis and exocytosis}

These processes involve the formation of a vesicle in order to transport substances into cells.

Endocytosis involves the invagination of the cell membrane around a substance, therefore absorbing it into the cell, and then pinching it off so that the portion of the membrane and its contents becomes an independent, intracellular vesicle (Figure 9). Endocytosis is subdivided into phagocytosis and pinocytosis.

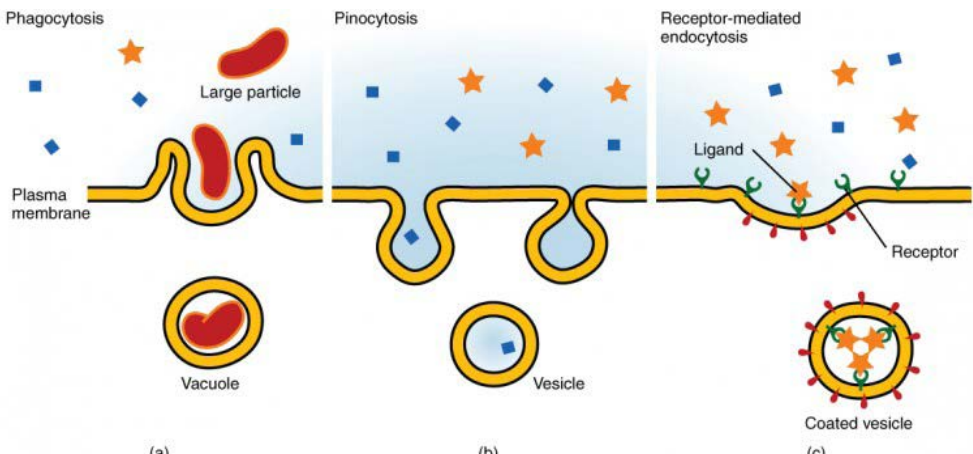

(a)

(b)

(c)

Figure 9: Three forms of endocytosis

Endocytosis is a form of active transport in which a cell envelopes extracellular material using its cell membrane. (a) In phagocytosis, which is relatively nonselective, the cell takes in a large particle. (b) In pinocytosis, the cell takes in small particles in fluid. (c) In contrast, receptorediated endocytosis is quite selective. When external receptors bind a specific ligand, the cell esponds by endocytosing the ligand. ${ }^{1}$

Phagocytosis is the endocytosis of larger particles. Immune cells engulf bacteria in this way, creating a vacuole in the leukocyte which eventually breaks them down and digests them. Fluid containing substances that are endocytosed but do not form a vacuole are done so through the process of pinocytosis. These two types of endocytosis are not necessarily very selective for the large extracellular particles that they 'ingest'. However, there are certain cells that regulate this process by receptor-mediated endocytosis. Receptors specific to certain substances will form receptor-ligand complexes that endocytose once completely bound and thus regulate that substance's admission into the cell. Typically, iron is regulated in this way when entering red blood cells. ${ }^{1,4}$

Exocytosis (Figure 10) is the process by which a vesicle of substances is secreted by an active calcium-dependant method when fusing with the plasma membrane. An example of this occurs in the cells of the stomach and pancreas where digestive enzymes and hormones are secreted into the body in this manner. ${ }^{1}$ 


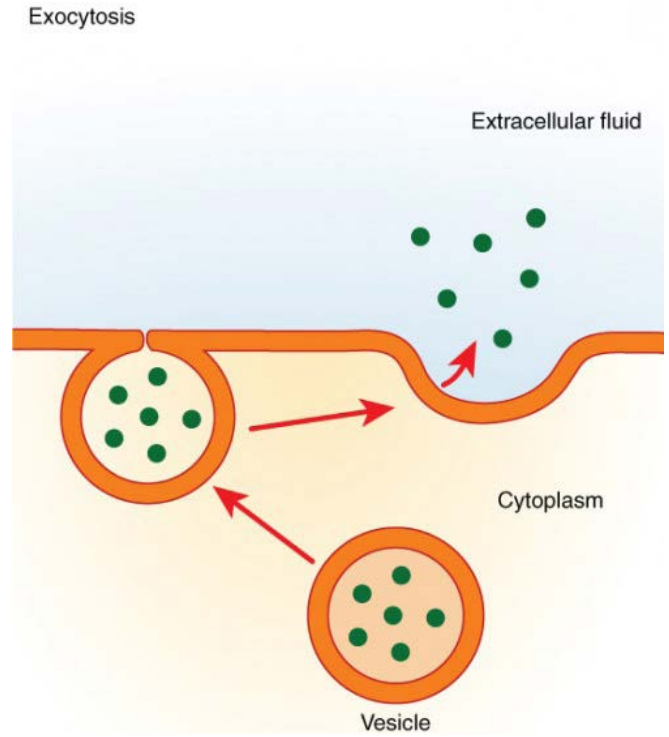

Figure 10: Exocytosis

Exocytosis is much like endocytosis in reverse. Material destined for export is packaged into a vesicle inside the cell. The membrane of the vesicle fuses with the cell membrane, and the contents are released into the extracellular space. ${ }^{1}$

\section{The Gibbs-Donnan effect}

The Gibbs-Donnan effect is described as the tendency of ions to unequally distribute themselves on either side of a semipermeable membrane when they are in the presence of non-diffusible ions. ${ }^{4}$

Gibbs-Donnan equilibrium/effect occurs when:

- On each side of the membrane, the solution is electrically neutral.

- The product of diffusible ions on one side of the membrane is equal to the product of diffusible ions on the other side of the membrane:

$$
\left[\mathrm{Na}^{+}\right] \mathrm{A} \times[\mathrm{Cl}] \mathrm{A}=\left[\mathrm{Na}^{+}\right] \mathrm{B} \times[\mathrm{Cl}] \mathrm{B}
$$

- The electrochemical gradients produced by unequal distribution of charged ions produces a transmembrane potential difference which can be calculated using the Nernst equation.

- The presence of an altered tonicity on one side of the membrane creates an osmotic diffusion gradient that attracts water into that compartment. ${ }^{4,8,9}$

The two main contributors to the Gibbs-Donnan effect are sodium and protein, because cell membranes are effectively impermeable to sodium due to the $\mathrm{Na}^{+} / \mathrm{K}^{+}$ATPase pump and because they are impermeable to protein in the face of high intracellular protein concentrations. ${ }^{4}$

Therefore, the Gibbs-Donnan effect is important for the maintenance of cell volume, plasma oncotic pressure and resting membrane potential. However, the mechanisms which maintain the resting membrane potential and the mechanisms of the Gibbs-Donnan effect are different phenomena:

- The Donnan equilibrium is a completely passive process, i.e. no active transporters are involved in maintaining this equilibrium.

- A Donnan equilibrium is an equilibrium, i.e. ion concentrations on either side of the barrier are static.

- If the Donnan equilibrium were to become fully established, the increase in intracellular ions would cause cells to swell due to the osmotic influx of water.

- At a Donnan equilibrium, the resting membrane potential would be only about $-20 \mathrm{mV}$. This potential would exist even if the membrane permeability for all ions was the same., ${ }^{8,9}$

In contrast the resting membrane potential requires different permeabilities for potassium and for sodium, and is maintained actively by constant $\mathrm{Na}^{+} / \mathrm{K}^{+}$ATPase activity, however biological membranes (especially those of nerve cells) are never at equilibrium, the Goldman-Hodgkin-Katz equation is usually a better choice for explaining their electrochemical behaviour. ${ }^{4,8,9}$

\section{Conflict of interest}

The author declares no conflict of interest.

\section{Funding source}

None.

\section{Ethical approval}

Not applicable.

\section{ORCID}

\section{Anamourlis (iD https://orcid.org/0000-0001-5509-2473}

\section{References}

1. Lumen Learning. Anatomy and Physiology. The Plasma Membrane Available from: https://courses.lumenlearning.com/nemcc-ap/chapter/ the-cellmembrane/. Accessed 12 October 2020.

2. Barrett KE, Barman SM, Boitano S, Brooks HL. Ganong's Review of Medical Physiology. 24th ed. McGraw Hill; 2012.

3. Chambers D, Huang C, Matthews G. Basic physiology for anaesthetists. Cambridge University Press; 2015. https://doi.org/10.1017/CBO9781139226394

4. Life in the Fast Lane. Part One. Cellular Physiology. Available from: https:// partone.litfl.com/the cell membrane.html\#id. Accessed 6 October 2020.

5. Kam P, Power I. Principles of physiology for the anaesthetists. 3rd ed. Hodder Education; 2012.

6. Deranged Physiology. Cellular Physiology. Mechanisms responsible for the cell resting membrane potential. Available from: https://derangedphysiology. $\mathrm{com} / \mathrm{main} / \mathrm{cicm}$-primary-exam/required-reading/cellular-physiology/ Chapter\%20118/mechanisms-responsible-cell-resting-membrane-potential. Accessed 12 October 2020

7. Eaton DC, Pooler JP. Vander's Renal Physiology. 6th ed (Revised). McGraw-Hill Education-Europe; 2004

8. PhysiologyWeb. Secondary Active Transport. Available from: https:// physiologyweb.com/lecture notes/membrane_transport/secondary active_transport.hml\#: :text=Secondary\%20active\%20transport\%20is\%20 a,against\%20a\%20concentration\%2Felectrochemical\%20gradient. Accessed 12 October 2020.

9. Deranged Physiology. Cellular Physiology. The Gibbs-Donnan Effect Available from: https://derangedphysiology.com/main/cicm-primary-exam/ requiredreading/cellular-physiology/Chapter\%20121/gibbs-donnan-effect. Accessed 12 October 2020 